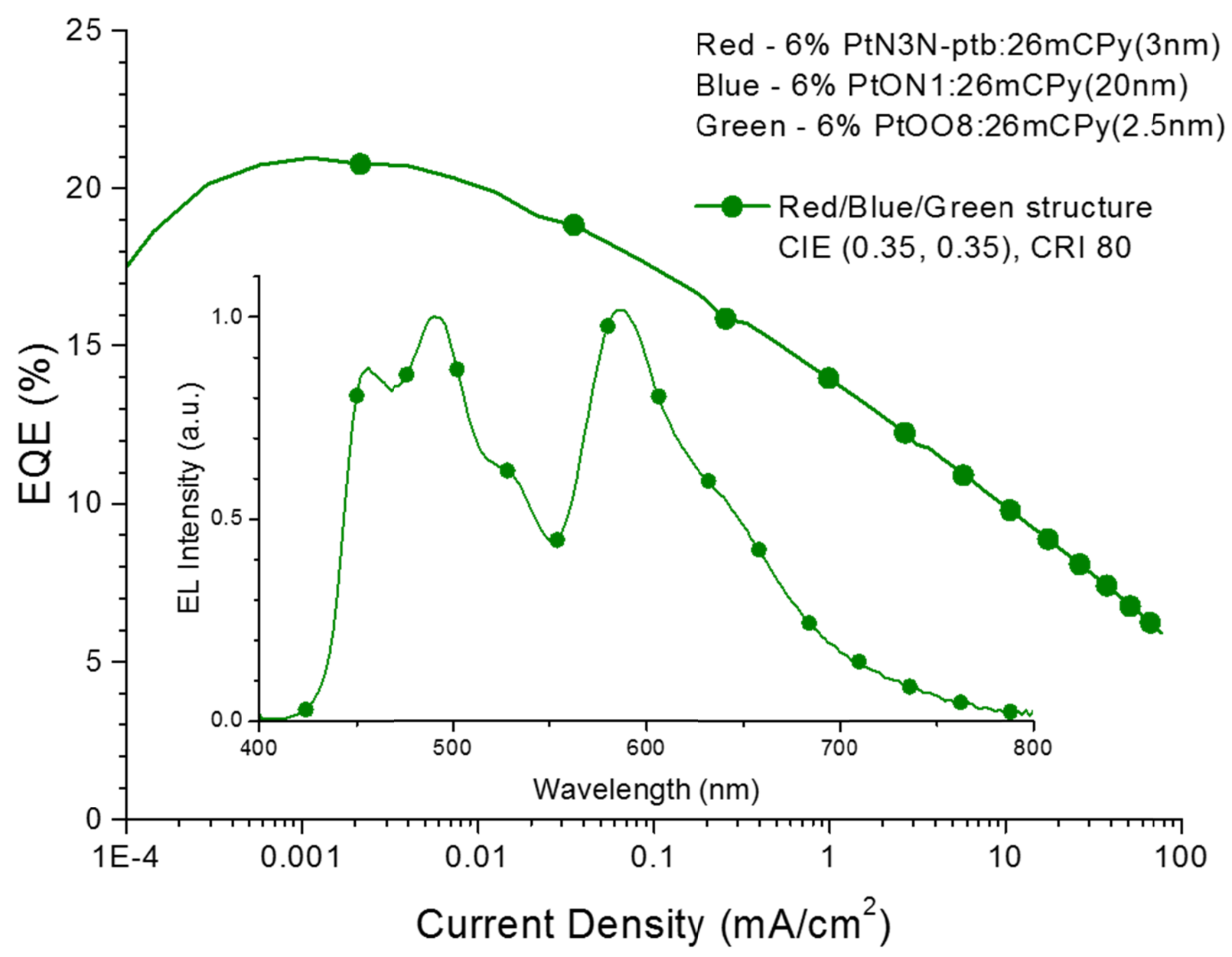




\title{
Efficient White OLEDs Employing Red, Green, and Blue Tetradentate Platinum Phosphorescent Emitters
}

\author{
Gregory E. Norby, Choong-Do Park, Barry O’Brien, Guijie Li, Liang Huang, and Jian Li*
}

Material Science and Engineering Program, Arizona State University, Tempe, AZ, 85281, USA.

* Corresponding author: Jian Li, Tel: (480) 727-8938, E-mail: jian.li.1@asu.edu

\begin{abstract}
:
The development of white organic light-emitting diodes (WOLEDs) employing three tetradentate platinum-based emitters, PtN3N-ptb (red), PtON1 (blue), and PtOO8 (green), is described. Spectral and electrical results show that localization of charge buildup, energy transfers between dopants, and concentration-dependence of spectral shape are all major factors in determining the overall color quality of a device. WOLEDs are fabricated by optimizing the thickness of emissive layers, the concentration of dopants, and the stacking order of emissive layers. Ultimately, the device employing 6\% PtN3N-ptb(red)/6\% PtON1(blue)/6\% PtOO8(green) stacking order of emissive layers demonstrates the Commission Internationale de L'Éclairage coordinates $(\mathrm{CIE})$ coordinates of $(0.35,0.35)$ and Color Rendering Index $(\mathrm{CRI})$ value of 80 , peak Power Efficiency (PE) of $41 \mathrm{~lm} / \mathrm{W}$, and maximum External Quantum Efficiency (EQE) of $21.0 \%$.
\end{abstract}

\section{Keywords: White OLEDs, Platinum emitters, Phosphorescence}

\section{Introduction}

In the effort to reduce energy use, the development of solid-state lighting technologies which are efficient, long-lasting, and made from environmentally benign materials are strongly desired. White Organic light-emitting devices (WOLEDs) show promise because they can be fabricated on a large number of substrate materials using various deposition methods that are 
compatible with high-throughput processing and can employ a variety of form factors such as large-area panels, curved surfaces, or even flexible devices. ${ }^{[1,5]}$ Through rigorous research efforts in the design of new emissive materials, device architectures, and out-coupling techniques, WOLEDs exceeding $100 \mathrm{~lm} / \mathrm{W}$ with a high color quality of light have been achieved. ${ }^{[5 c]}$

Two parameters describe the color quality of a white light source: Commission Internationale de L'Éclairage coordinates (CIE) and the Color Rendering Index (CRI) value, where CIE coordinates of $(0.33,0.33)$ is considered "pure white" and a CRI value as close to 100 as possible is desired. ${ }^{[2]}$ There is a wide range of approaches to achieve high color quality with white organic light emitting diodes. These include multiple emissive materials within a device, striped regions of red, green, and blue emission, combination of fluorescent and phosphorescent emission, or the combination of monomer and excimer emission from square planar metal complexes. ${ }^{[3,4,5 b, 6,7]}$ Of these various options, the combination of three emissive dopants has typically been favored due to the ability to obtain a broad spectrum of high-quality white light by controlling the relative contribution of each emissive material to optimize CIE coordinates and CRI value. In particular, the use of multiple emissive layers (EMLs) to separate the red, green and blue emissive dopants has seen success in minimizing interaction between emitters to achieve balanced color quality and high efficiencies. ${ }^{[4,5 c,]}$ However, the efficiency, color quality, and stability of these devices are still far from commercial application. ${ }^{[5 c, 7 b, 8,9]}$ Therefore more improvements must be made in both materials design and device architectures, especially through the development of new emissive materials.

To date, much of the research into emissive materials has focused on iridium-based complexes, for which external quantum efficiencies (EQE) of $25 \%$ and higher have been reported for emission ranging across the whole visible spectrum. ${ }^{[8]}$ Iridium complexes also tend 
to have a shorter radiative decay time, which makes it easier to achieve higher efficiency with low roll-off. Platinum complexes, on the other hand, have received significantly less attention for WOLED applications, despite recent reports of performance and color purity equivalent or superior to their iridium analogs ${ }^{[9]}$ The square planar ligand coordination of Pt complexes also allows the design of rigid tetradentate metal complexes, which shortens radiative decay process and increases the photoluminescence quantum efficiency. Thus, due to their potential for high performance and desirable color quality, platinum complexes for WOLEDs should be vigorously investigated as alternative emissive materials for the lighting applications. In this report, the development of WOLEDs utilizing red, green, and blue Pt-based phosphorescent emitters is described. Furthermore, the charge transport and energy transfer processes for WOLEDs employing multiple platinum-based emitters are studied. Ultimately, a WOLED with the CRI value of 80 , CIE coordinates of $(0.35,0.35)$, maximum external quantum efficiency (EQE) of $21.0 \%$, and maximum power efficiency (PE) of $41 \mathrm{~lm} / \mathrm{W}$ is obtained. Such a performance is comparable to the state-of-the-art of WOLEDs employing multiple Ir-based phosphorescent emitters. ${ }^{[9]}$ To the best of author's knowledge, this work is the first report of multiple Pt-based emitting layer WOLED that demonstrates the peak EQE of 21.0\%.

\section{Materials and Methods}

Materials:

Three tetradentate Pt complexes of PtN3N-ptb (red), PtOO8 (green), and PtON1 (blue) are selected to cover a broad range of visible spectrum. ${ }^{[10]}$ Synthetic methods for the three Pt complexes are described in the Supporting Information. The general structure used for all OLEDs fabricated is: ITO/ HATCN(10nm)/ NPD(40nm)/ TAPC(10nm)/ EML/ DPPS(10nm)/ $\mathrm{BmPyPB}(40 \mathrm{~nm}) / \mathrm{LiF}(1 \mathrm{~nm}) / \mathrm{Al}(100 \mathrm{~nm})$. Here, HATCN is 1,4,5,8,9,11-hexaazatriphenylene- 
hexacarbonitrile. ${ }^{[11]}$ NPD is N,N'-diphyenyl-N,N'-bis(1-naphthyl)-1,1'-biphenyl-4,4''-diamine. TAPC is di-[4-(N,N-di-toylyl-amino)-phyenyl] cyclohexane. ${ }^{[12 c]}$ The EML is an emissive layer consisting of emissive materials doped in $26 \mathrm{mCPy}, 2,6$-bis(N-carbazolyl) pyridine. ${ }^{[12 \mathrm{~b}]} \mathrm{DPPS}$ is diphenyl-bis[4-(pyridin-3-yl)phenyl]silane. ${ }^{[12 a]}$ BmPyPB is 1,3-bis[3, 5-di(pyridin-3yl)phenyl]benzene. ${ }^{[13]}$ Further material properties and synthesis methods are described in Supplemental Information.

Device Fabrication and Characterization:

OLEDs are fabricated using a thermal evaporation process, depositing the materials onto prepatterned ITO/glass substrates. Substrates are cleaned by hand washing and sonication in water, and then acetone, then isopropyl alcohol. Deposition occurred by vacuum thermal evaporation in a custom-built chamber by Trovato Manufacturing Inc. at pressures ranging from $10^{-8}-10^{-7}$ torr, at nominal rates of up to $1 \AA / \mathrm{s}$. The ITO anodes and aluminum cathodes are each patterned $2 \mathrm{~mm}$ wide perpendicular to each other, resulting in a device area of $0.04 \mathrm{~cm}^{2}$. Current Density/ Voltage/ Luminance (JVL) behavior is characterized by a Keithley 2400 SourceMeter and a Newport 818-UV photodiode. Irradiance spectra are captured using a HR4000 spectrometer (Ocean Optics), $1000 \mu \mathrm{m}$ fiber optic cable, calibrated using an LS-1-CAL tungsten halogen lamp (Ocean Optics).

\section{Results \& Discussion}

Monochromatic devices using each individual emitter are fabricated in a general structure of ITO/ HATCN/ NPD/ TAPC/ 26mCPy: x\% emitter (25nm)/ DPPS/ BmPyPB/ LiF/ Al. The plots of electroluminescence (EL) spectra as a function of wavelength, plots of EQE vs. current density, and the corresponding molecular structures of each emitter are shown in Figure 1. Additionally, the current density-voltage (J-V) curves are shown in Figure S1. Peak external 
quantum efficiency (EQE) values of $17 \%, 23 \%$, and $21 \%$ are achieved for $2 \%$ PtN3N-ptb, $6 \%$ PtOO8, and 6\% PtON1 doped devices respectively. The device with 6\% PtON1 doping concentration exhibits a deep blue emission spectrum, which covers much of the blue region necessary to achieve high CRI value. The device employing $6 \%$ PtOO8 doping concentration shows an emission peak at $498 \mathrm{~nm}$ and a secondary emission peak at $530 \mathrm{~nm}$ to provide an appropriate green color. The emission spectra of the 2\% PtN3N-ptb doped device peaks at 588 $\mathrm{nm}$ but drops off sharply, missing a significant portion of the red spectrum beyond $650 \mathrm{~nm}$. Upon increasing the dopant concentration of PtN3N-ptb from $2 \%$ to $6 \%$, its spectrum is broadened and red-shifted to a peak wavelength of $593 \mathrm{~nm}$, due to a rise in the vibronic emission sideband. This vibronic emission is desirable to include more of the red spectrum for a high-quality white light. A linear combination of the emission intensities of the three emitters suggests that the use of $2 \%$ PtN3N-ptb emissive layer would lead to a maximum possible CRI value of 79 , while the use of 6\% PtN3N-ptb emissive layer would lead to a maximum possible CRI value of 82 , neglecting interactions between the emitters or optical interference effects. ${ }^{[2,14]}$ However, the increase in the dopant concentration of PtN3N-ptb based red OLED decreases the peak EQE to 15\%. Thus, the PtN3N-ptb concentration poses a trade-off between optimizing the device efficiency and the color quality. 

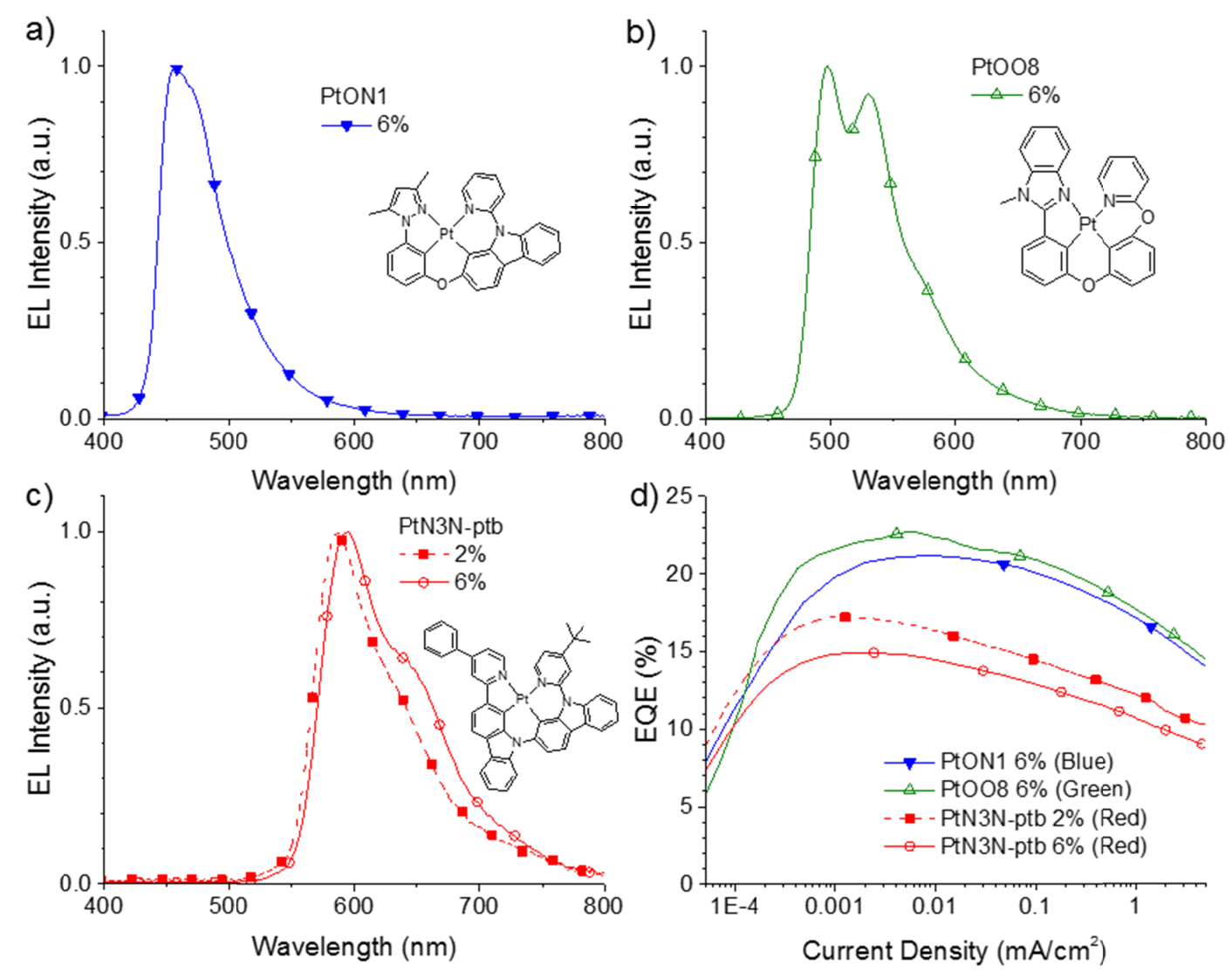

Figure 1. a)-c) EL Spectra as a function of wavelength and d) plots of EQE vs. current density of OLEDs with a device structure of ITO/ HATCN/ NPD/ TAPC/ Emitter: 26mCPy(25nm)/ DPPS/ BmPyPB/ LiF/ Al. The emitters used are a) PtON1 (blue), b) PtOO8 (green), and c) PtN3N-ptb (red). Inset figures are molecular structures of emitters used. The corresponding molecular structures of each emitter are presented in the inset of Figures.

WOLEDs are prepared by replacing the emissive layer in the aforementioned general structure with multiple stacks of single-doped red, green, or blue emitters. In multilayer WOLEDs, it is necessary to tune the contribution of emitters to produce a balanced emission spectrum. Two major order-dependent factors affect the relative output between emitters: variation of recombination zone within the emissive layers, and energy transfer between dopants. Without a perfect charge carrier balance, the recombination zone is likely to be localized adjacent to one of the charge blocking layers. ${ }^{[15]}$ As a result, whichever dopant is present in the 
recombination region is predicted to have the majority of contributions to the EL spectrum of a device. As shown in the EL spectra in Figure 2, the device with TAPC/ 6\% PtN3N-ptb:26mCPy/ 6\% PtON1:26mCPy/ DPPS structure exhibits a strong blue emission from the PtON1 complex. However, when a PtN3N-ptb layer is adjacent to the hole blocking layer of DPPS, the PtN3N-ptb become a major contributor to the EL spectrum even if the thickness of the PtN3N-ptb layer $(3 \mathrm{~nm})$ is much thinner than that of the PtON1 layer $(20 \mathrm{~nm})$. This result suggests that for a WOLED with three emissive layers, the emitter deposited next to DPPS will likely exhibit the strongest emission due to the localized recombination zone formed near to the hole blocking layer of DPPS. Interestingly, however, the lower EQE (16\%) of 6\% PtN3N-ptb doped red OLED shown in Figure 1 (d) appears not to translate to the multiple emissive layer WOLEDs employing 6\% PtN3N-ptb:26mCPy and 6\% PtON1:26mCPy emissive layers. As illustrated in Figure 2, both devices show similar peak EQE values above 22\%, albeit with sharp roll-off at higher current densities. 


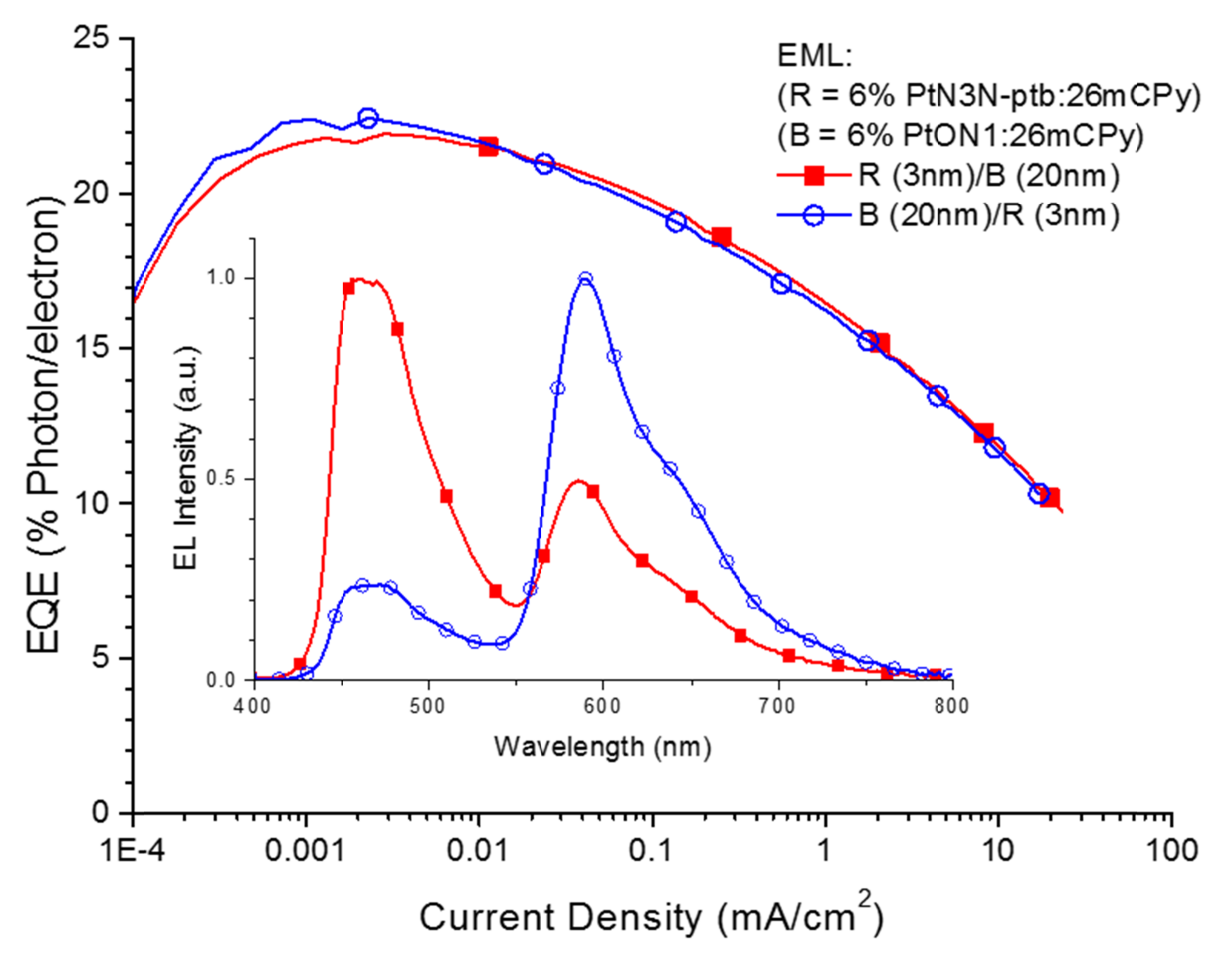

Figure 2. Plots of EQE vs. current density and the EL Spectra (inset) of WOLEDs with the device structure of ITO/ HATCN/ NPD/ TAPC/ EML/ DPPS/ BmPyPB/ LiF/ Al, where EML is the layers of $6 \%$ PtN3N-ptb:26mCPy $(3 \mathrm{~nm}) / 6 \%$ PtON1:26mCPy $(20 \mathrm{~nm})$ or 6\% PtON1 (20 $\mathrm{nm}) / 6 \%$ PtN3N-ptb: 26mCPy (3 nm).

WOLEDs are fabricated with three emissive layers in the stacking order of $6 \%$ PtON1:26mCPy/ 6\% PtOO8:26mCPy/ 2\% PtN3N-ptb:26mCPy, termed Structure I: ITO/ HATCN/ NPD/ TAPC/ 6\% PtON1: 26mCPy (20nm)/ 6\% PtOO8: 26mCPy (x)/ 2\% PtN3N-ptb: 26mCPy (3nm)/ DPPS/ BmPyPB/ LiF/ Al, where $\mathrm{x}$ is 2, 3, $4 \mathrm{~nm}$. In Structure I, a thin (3nm), lightly doped (2\%) PtN3N-ptb layer is deposited nearest to the DPPS interface, while a 6\% PtOO8 doped layer is inserted between PtON1 and PtN3N-ptb doped layers to add the green portion of the visible spectrum. In order to examine the effect of the PtOO8 layer on the device performance, the thickness of $6 \%$ PtOO8 layer is varied from $2 \mathrm{~nm}$ to $4 \mathrm{~nm}$, while the thicknesses of PtON1 and PtN3N-ptb doped layers are fixed to 20nm and 3nm, respectively. The resulting EL spectra and EQE vs. current density curves are given in Figure 3, and the current density- 
voltage data are shown in Figure S3. The EQE and PE of the devices show minimal variation with increasing the thickness of the $6 \%$ PtOO8 doped layer with peak EQE ranging from 20.8\% to $21.2 \%$, and peak PE of 43 to $45 \mathrm{~lm} / \mathrm{W}$. Despite the high overall efficiencies, there is a minimal PtOO8 contribution to the emission spectrum, resulting an unsatisfactory CRI value of 70 . The low contribution of PtOO8 layer is likely due to a high rate of energy transfer from PtOO8 complex $\left(\mathrm{E}_{\mathrm{T}}=2.5 \mathrm{eV}\right)$ to nearby PtN3N-ptb complex $\left(\mathrm{E}_{\mathrm{T}}=2.1 \mathrm{eV}\right) .{ }^{[\mathrm{a}]}$ Moreover, as the thickness of the PtOO8 layer increases from $2 \mathrm{~nm}$ to $4 \mathrm{~nm}$, the blue emission of PtON1 complex decreases, and then results in the change of the CIE coordinates from $(0.38,0.35)$ to $(0.42,0.39)$ and the decrease of CRI value from 70 to 67 , respectively. The decrease in the PtON1 emission can be explained regarding the depth of a recombination zone. When a recombination zone is formed at an interface between an emissive layer and a charge blocking layer, typically, the depth of a recombination zone is known to be less than $10 \mathrm{~nm}$. Thus, when the recombination zone is formed at the interface between the PtN3N-ptb emissive layer and the hole blocking layer of DPPS, with increasing the thickness of PtOO8 layer, the PtON1 layer is pushed away from the recombination zone, in turn, results in the decreased emission of PtON1 complex, as illustrated in Figure 3. 


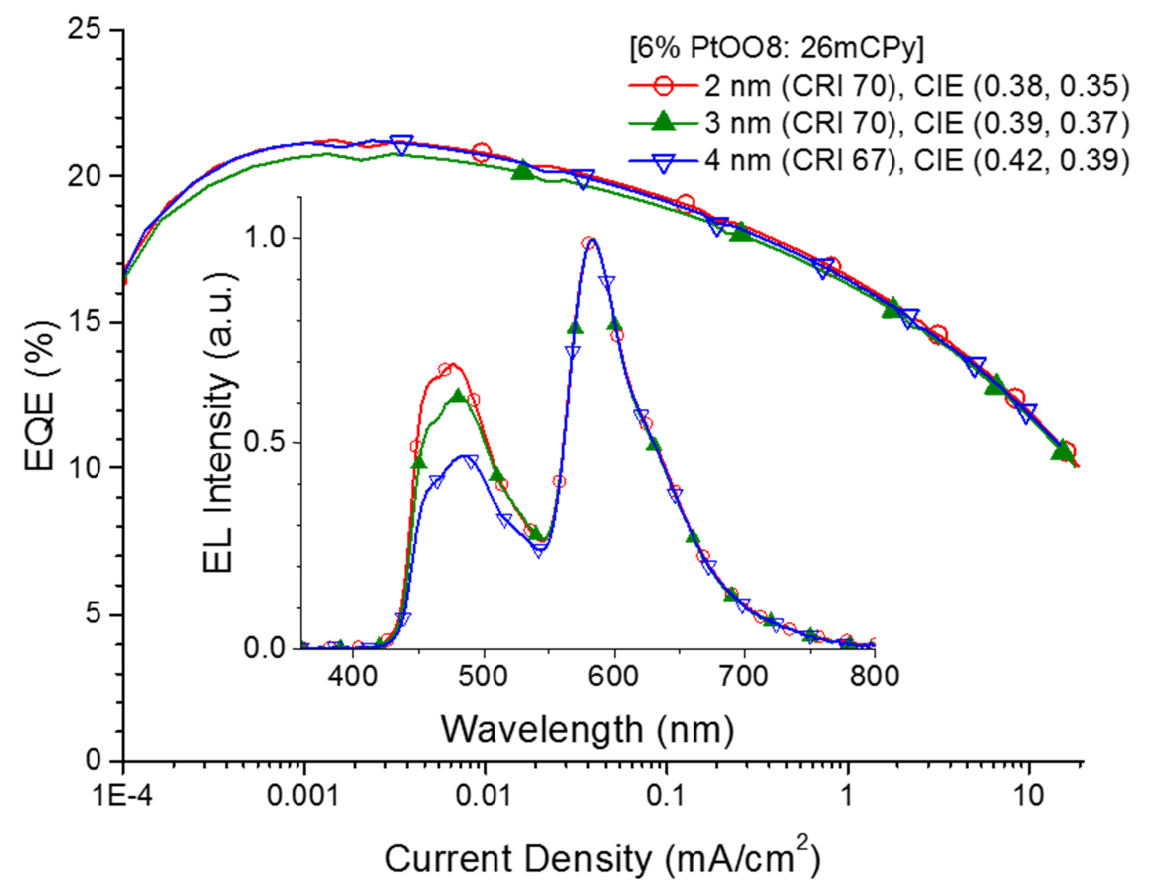

Figure 3. EL spectra as a function of wavelength (inset) and the plots of EQE vs. current density of WOLEDs with a device structure of ITO/ HATCN/ NPD/ TAPC/ 6\% PtON1: 26mCPy (20nm)/ 6\% PtOO8: 26mCPy (x nm)/ 2\% PtN3N-ptb: 26mCPy (3 nm)/ DPPS/ BmPyPB/ LiF/ $\mathrm{Al}$. The thickness of 6\% PtOO8: $26 \mathrm{mCPy}$ layer was varied in the range of $2-4 \mathrm{~nm}$. The EL spectra are normalized to the PtN3N-ptb emission peak.

To account for the strong energy transfer from PtOO8 to PtN3N-ptb, their respective emitting layers were separated by inserting a thick PtON1 doped emissive layer between a PtOO8 doped layer and a PtN3N-ptb doped layer with a new device Structure II: ITO/ HATCN/ NPD/ TAPC/ x\% PtN3N-ptb: 26mCPy(3 nm)/ 6\% PtON1: 26mCPy(20 nm)/ 6\% PtOO8: 26mCPy(2.5nm)/ DPPS/ BmPyPB/ LiF/ Al. In this configuration, the thick (20 nm), highbandgap PtON1 doped emissive layer will prevent the energy transfer from PtOO8 to PtN3N-ptb complex, allowing more contribution of PtOO8 complex to the EL spectrum. The EL Spectra and EQE data for the devices employing Structure II are shown in Figure 4 (a). General spectral results and device efficiencies at operational conditions for all the devices studied are also summarized in Table 1. The EL spectra show that, by separating the PtOO8 and PtN3N-ptb 
doped layers with the thick (20nm) PtON1 layer, the energy transfer from PtOO8 to PtN3N-ptb is substantially reduced allowing improved contribution of PtOO8 to the EL spectrum compared to that of the devices with Structure I shown in Figure 3. Moreover, as the dopant concentration of PtN3N-ptb increases from 2\% to 6\%, both PtON1 and PtN3N-ptb emission increases relative to the PtOO8 emission. The increased PtN3N-ptb emission can be explained by the increased dopant concentration of PtN3N-ptb complex that allows trapping more charges to form redemitting excitons and subsequently results in the stronger red emission. ${ }^{[10,12 b]}$ The increase in PtON1 emission is also attributed to the increase in the dopant concentration of PtN3N-ptb that causes a shift of a recombination zone to the interface between PtN3N-ptb and PtON1 layer. The CIE coordinates of $(0.32,0.40)$ and CRI value of 76 for the device with Structure II device containing a $2 \%$ PtN3N-ptb doped layer are much improved from the devices with Structure I, as summarized in Table 1. The devices employing Structure II maintains a high maximum EQE of $21.0 \%$ and a maximum power efficiency of $41 \mathrm{~lm} / \mathrm{W}$. Upon increasing the PtN3N-ptb dopant concentration from $2 \%$ to $6 \%$, the CIE coordinates shifted from $(0.32,0.40)$ to $(0.35,0.35)$, and the CRI value is increased from 76 to 80 , respectively. Maximum EQE for the device with $6 \%$ PtN3N-ptb doped layer remained at 21.0\%, but the high roll-off of EQE is observed with increasing current density, dropping to $11.5 \%$ at $1000 \mathrm{~cd} / \mathrm{m}^{2}$ compared to $13.5 \%$ for the device employing 2\% PtN3N-ptb doped layer. Also, the device containing a 6\% PtN3N-ptb doped layer showed more significant roll-off with increasing the current density, as illustrated in Figure 4 (a). This higher roll-off is related to the increased level of trapped charges and excitons with increasing the dopant concentration. ${ }^{[18]}$ 

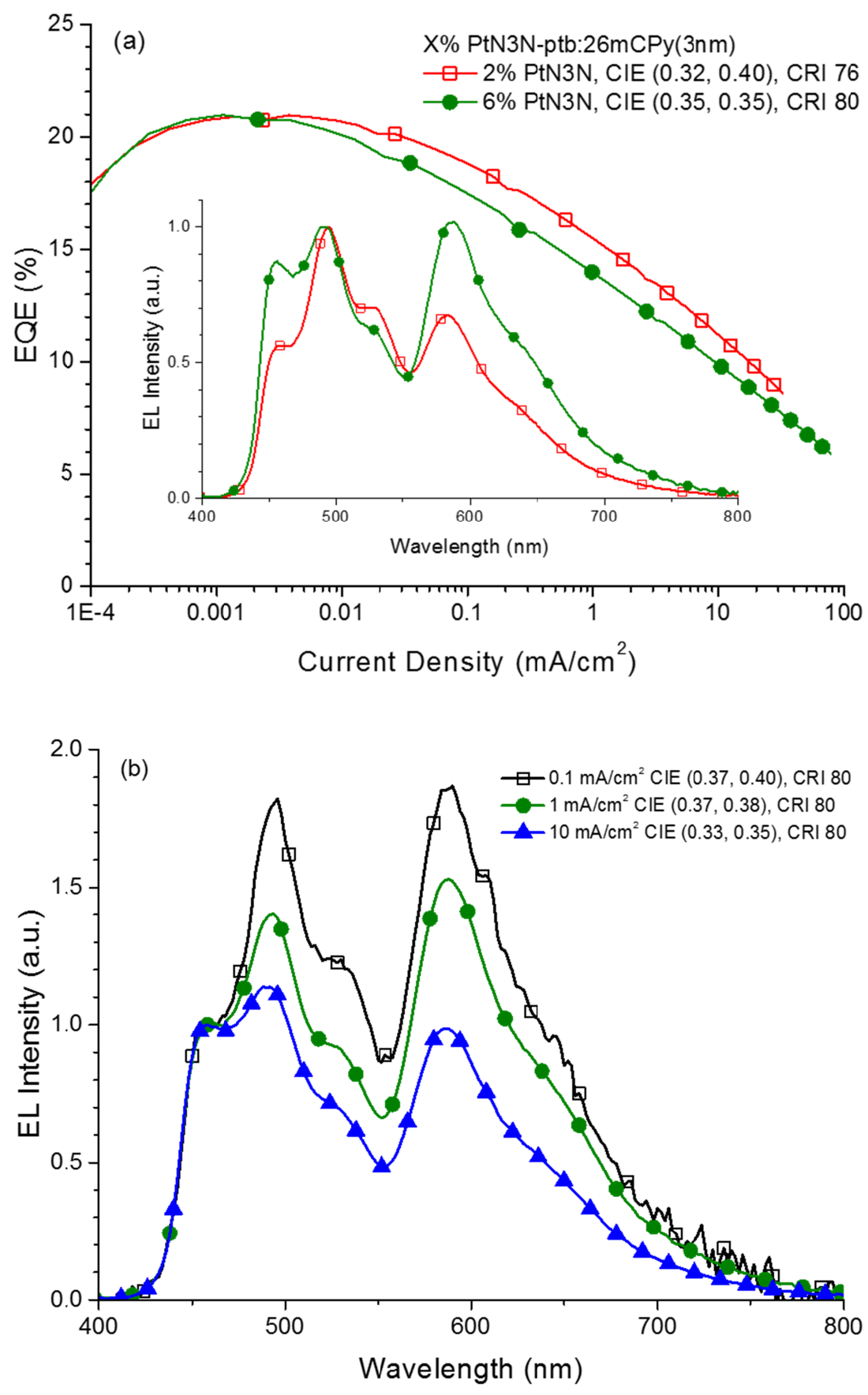

Figure 4. (a) EL Spectra (inset) as a function of wavelength and the plots of EQE vs current density of WOLEDs with a device structure of ITO/ HATCN/ NPD/ TAPC/ x\% PtN3N-ptb: 26mCPy $(3 \mathrm{~nm}) / 6 \%$ PtON1: 26mCPy $(20 \mathrm{~nm}) / 6 \%$ PtOO8: 26mCPy $(2.5 \mathrm{~nm}) / \mathrm{DPPS} / \mathrm{PmPyPB} /$ $\mathrm{LiF} / \mathrm{Al}$. PtN3N-ptb concentration $(\mathbf{x} \%)$ was varied between $2 \%$ and $6 \%$. The spectra are normalized to the PtOO8 emission peak; (b) EL Spectra of a WOLED with a device structure of ITO/ HATCN/ NPD/ TAPC/ 6\% PtN3N-ptb: 26mCPy (3 nm)/ 6\% PtON1: 26mCPy (20 nm)/ 6\% PtOO8: 26mCPy (2.5nm)/ DPPS/ PmPyPB/ LiF/ Al at various current densities ranging from 0.1 $-10 \mathrm{~mA} / \mathrm{cm}^{2}$. The spectra are normalized to the PtON1 emission peak at $455 \mathrm{~nm}$. 
Table 1. Summary of device performances for color quality (CIE), color balance (CRI), EQE $(\%)$, current efficiency (cd/A), and luminous efficacy $(\mathrm{lm} / \mathrm{W})$. Here "B" denotes PtON1, "G" denotes PtOO8, and "R" denotes PtN3N-ptb.

\begin{tabular}{|c|c|c|c|c|c|c|c|c|c|c|c|}
\hline \multirow{2}{*}{$\begin{array}{l}\text { Struc- } \\
\text { ture }\end{array}$} & \multirow{2}{*}{$\begin{array}{c}\text { R } \\
\text { Conc. } \\
\text { (wt \%) }\end{array}$} & \multicolumn{2}{|c|}{$\begin{array}{c}\text { Thickness } \\
\text { (nm) }\end{array}$} & \multirow{2}{*}{$\operatorname{CIE}(x, y)^{*}$} & \multirow{2}{*}{ CRI' } & \multicolumn{3}{|c|}{$100 \mathrm{~cd} / \mathrm{m}^{2}$} & \multicolumn{3}{|c|}{$1000 \mathrm{~cd} / \mathrm{m}^{2}$} \\
\hline & & $\mathrm{R}$ & $\mathrm{G}$ & & & $\begin{array}{l}\eta_{\mathrm{EQE}} \\
(\%)\end{array}$ & $\begin{array}{c}\eta_{\mathrm{A}} \\
\text { (cd/A) }\end{array}$ & $\begin{array}{c}\eta_{\mathrm{p}} \\
(\mathrm{lm} / \mathrm{W})\end{array}$ & $\begin{array}{l}\eta_{\mathrm{EQE}} \\
(\%)\end{array}$ & $\begin{array}{c}\eta_{\mathrm{A}} \\
(\mathrm{cd} / \mathrm{A})\end{array}$ & $\begin{array}{c}\eta_{\mathrm{p}} \\
(\mathrm{lm} / \mathrm{W})\end{array}$ \\
\hline \multirow{3}{*}{$\begin{array}{c}\mathrm{I} \\
\text { (BGR) }\end{array}$} & 2 & 3 & 2 & $(0.38,0.35)$ & 70 & 18.4 & 40 & 25 & 14.6 & 31 & 15.8 \\
\hline & 2 & 3 & 3 & $(0.39,0.37)$ & 70 & 18.0 & 40 & 25 & 14.4 & 32 & 15.9 \\
\hline & 2 & 3 & 4 & $(0.42,0.39)$ & 67 & 18.3 & 42 & 26 & 14.5 & 33 & 16.0 \\
\hline \multirow{2}{*}{$\begin{array}{c}\text { II } \\
\text { (RBG) }\end{array}$} & 2 & 3 & 2.5 & $(0.32,0.40)$ & 76 & 17.6 & 41 & 28 & 13.5 & 31 & 16.9 \\
\hline & 6 & 3 & 2.5 & $(0.35,0.35)$ & 80 & 15.8 & 32 & 24 & 11.5 & 24 & 14.0 \\
\hline
\end{tabular}

CIE and CRI were measured at $\mathrm{J}=1 \mathrm{~mA} / \mathrm{cm}^{2}$.

To further understand the efficiency roll-off and the color stability of the device with Structure II, EL spectra are recorded for Structure II device with a 6\% PtN3N-ptb doped layer at various driving current densities in the range of $0.1-10 \mathrm{~mA} / \mathrm{cm}^{2}$. The resulting spectra, in Figure 4(b), demonstrate a significant increase in PtON1's contribution with increasing current density, relative to PtOO8 and PtN3N-ptb emission. At low current densities, charges are trapped in the PtN3N-ptb and PtOO8 doped layers, and primary recombination occurs near the two blocking layers. As the current density increases, charge accumulation in the PtN3N-ptb-doped layer, as well as saturation of PtOO8 sites, shifts the recombination zone deeper into the PtON1doped emissive layer. Furthermore, the charge and exciton saturation in the PtN3N-ptb-doped layer lead to high rates of non-radiative decay via triplet-triple annihilation and a significant loss in quantum efficiency. As a result, the emission from PtN3N-ptb and PtOO8 are reduced relative to PtON1 emission. This relationship between current density and the quantum efficiency is reflected in the EQE curves by a significant efficiency roll-off. 
Despite the enhancement made for the device efficiencies, the further improvement of the color quality of the devices is limited by this materials system due to the significant gap in the spectrum between the PtON1 (blue) and PtN3N-ptb (red) emission spectra. The replacement of PtOO8 with a yellow emitter and further development of efficient and broad red emitters would yield an even better color quality, and CRI values of 85 to 90 should be within reach. Similarly, further improvement in efficiency is limited by the current structure's use of thin EMLs for PtOO8 and PtN3N-ptb, because these layers trap large quantities of charge carriers and excitons, leading to increased triplet-triplet annihilation losses. Further optimization of the device architecture to reduce charge and exciton build-up, the selection of more efficient transport materials, and the use of advanced outcoupling techniques should yield improved device efficiency, reduced device efficiency roll-off at high current density, and enhanced color stability in future WOLED development.

\section{Conclusion}

The effects of local recombination rate, dopant-to-dopant energy transfer, and charge trapping on color balance in triple platinum emitter WOLEDs have been investigated. Various thicknesses, dopant concentrations, and emitting layer stacking orders are studied to control precisely the charge balance and energy transfer processes occurring within the devices. Spectral and electrical data indicate that significant energy transfers occur from PtOO8 to PtN3N-ptb for the devices employing PtON1(blue)/PtOO8(green)/PtN3N-ptb(red) structure. By separating the PtOO8 and PtN3N-ptb emissive layers with a thick PtON1 doped blue emissive layer to inhibit the energy transfer, optimal color balance and quantum efficiency are achieved. Ultimately the device with 6\% PtN3N-ptb: 26mCPy (red)/6\% PtON1: 26mCPy (blue)/ 6\% PtOO8: 26mCPy (green) stacking order of emissive layers demonstrates the peak EQE of 21.0\%, CRI value of 80, 
and CIE coordinates of $(0.35,0.35)$. To the best of authors knowledge, this is the first report of a multiple Pt-based RGB emitting layer WOLED that demonstrates the peak EQE of $21.0 \%$. However, for the improved performance of WOLEDs employing multiple Pt-based RGB emitters, it is required to maintain continued emitter development and to optimize device architecture with the advanced outcoupling techniques.

\section{Acknowledgements}

The authors thank the Universal Display Corporation, National Science Foundation (CHE0748867), the Department of Energy (DE-EE0007090), and Flexible Display Center for partial support of this work.

\section{References:}

[1] a) M.C. Gather, A. Köhnen, K. Meerholz, White Organic Light-Emitting Diodes, Advanced Materials. 23 (2011) 233-248. doi:10.1002/adma.201002636; b) L. Xiao, Z. Chen, B. Qu, J. Luo, S. Kong, Q. Gong, et al., Recent Progresses on Materials for Electrophosphorescent Organic Light-Emitting Devices, Advanced Materials. 23 (2011) 926-952.

doi:10.1002/adma.201003128; c) H. Sasabe, J. Kido, Development of high-performance OLEDs for general lighting, Journal of Materials Chemistry C. 1 (2013) 1699. doi:10.1039/c2tc00584k;

d) H. Sasabe, J. Kido, Recent Progress in Phosphorescent Organic Light-Emitting Devices: Recent Progress in Phosphorescent Organic Light-Emitting Devices, European Journal of Organic Chemistry. 2013 (2013) 7653-7663. doi:10.1002/ejoc.201300544.

[2] a) Commission Internationale de l'Eclairage, Colour rendering (TC 1-33 closing remarks), Vienna (Austria): CIE. Publication No. 135/2:1999. (1999); b) Commission 
Internationale de l'Eclairage, Method of measuring and specifying color rendering properties of light sources, Vienna (Austria): CIE. Publication No. 13.3:1995. (1995) 16.

[3] a) Junji Kido, Masato Kimura, Katsutoshi Nagai, Multilayer white light-emitting organic electroluminescent device, Science. 267 (1995) 1332-1334; b) M.C. Gather, A. Köhnen, A. Falcou, H. Becker, K. Meerholz, Solution-Processed Full-Color Polymer Organic Light-Emitting Diode Displays Fabricated by Direct Photolithography, Advanced Functional Materials. 17 (2007) 191-200. doi:10.1002/adfm.200600651.

[4] a) Y.-L. Chang, Y. Song, Z. Wang, M.G. Helander, J. Qiu, L. Chai, et al., Highly Efficient Warm White Organic Light-Emitting Diodes by Triplet Exciton Conversion, Advanced Functional Materials. 23 (2013) 705-712. doi:10.1002/adfm.201201858; b) H. Sasabe, J. Takamatsu, T. Motoyama, S. Watanabe, G. Wagenblast, N. Langer, et al., High-Efficiency Blue and White Organic Light-Emitting Devices Incorporating a Blue Iridium Carbene Complex, Advanced Materials. 22 (2010) 5003-5007. doi:10.1002/adma.201002254; c) R. Wang, D. Liu, H. Ren, T. Zhang, H. Yin, G. Liu, et al., Highly Efficient Orange and White Organic LightEmitting Diodes Based on New Orange Iridium Complexes, Advanced Materials. 23 (2011) 2823-2827. doi:10.1002/adma.201100302; d) S. Chen, G. Tan, W.-Y. Wong, H.-S. Kwok, White Organic Light-Emitting Diodes with Evenly Separated Red, Green, and Blue Colors for Efficiency/Color-Rendition Trade-Off Optimization, Advanced Functional Materials. 21 (2011) 3785-3793. doi:10.1002/adfm.201100895; e) Q. Wang, J. Ding, D. Ma, Y. Cheng, L. Wang, F. Wang, Manipulating Charges and Excitons within a Single-Host System to Accomplish Efficiency/CRI/Color-Stability Trade-off for High-Performance OWLEDs, Advanced Materials. 21 (2009) 2397-2401. doi:10.1002/adma.200803312; f) Y. Zhao, J. Chen, D. Ma, Realization of high-efficiency orange and white organic light emitting diodes by introducing an ultra-thin 
undoped orange emitting layer, Applied Physics Letters. 99 (2011) 163303.

doi:10.1063/1.3654150. g) Wook Song, Inho Lee, and Jun Yeob Lee, “Host Engineering for High Quantum Efficiency Blue and White Fluorescent Organic Light-Emitting Diodes,”

Advanced Materials 27 (29), 4358-4363. h) Mounggon Kin and Jun Yeob Lee, "Engineering the Substitution Position of Diphenylphosphine Oxide at Carbazole for Thermal Stability and High External Quantum Efficiency Above 30\% in Blue Phosphorescent Organic Light-Emitting Diodes," Advanced Functional Materials 24 (2), 4164-4169.

[5] a) J. van den Brand, J. de Baets, T. van Mol, A. Dietzel, Systems-in-foil - Devices, fabrication processes and reliability issues, Microelectronics Reliability. 48 (2008) 1123-1128. doi:10.1016/j.microre1.2008.06.030; b) J. Kido, K. Hongawa, K. Okuyama, K. Nagai, White light-emitting organic electroluminescent devices using the poly(N-vinylcarbazole $)$ emitter layer doped with three fluorescent dyes, Applied Physics Letters. 64 (1994) 815.

doi:10.1063/1.111023; c) S. Reineke, F. Lindner, G. Schwartz, N. Seidler, K. Walzer, B. Lüssem, et al., White organic light-emitting diodes with fluorescent tube efficiency, Nature. 459 (2009) 234-238. doi:10.1038/nature08003.

[6] a) V. Adamovich, J. Brooks, A. Tamayo, A.M. Alexander, P.I. Djurovich, B.W. D'Andrade, et al., High-efficiency single dopant white electrophosphorescent light emitting diodes, New Journal of Chemistry. 26 (2002) 1171-1178. doi:10.1039/b204301g; b) T. Fleetham, J. Ecton, Z. Wang, N. Bakken, J. Li, Single-Doped White Organic Light-Emitting Device with an External Quantum Efficiency Over 20\%, Advanced Materials. 25 (2013) 25732576. doi:10.1002/adma.201204602.

[7] a) Y. Sun, N.C. Giebink, H. Kanno, B. Ma, M.E. Thompson, S.R. Forrest, Management of singlet and triplet excitons for efficient white organic light-emitting devices, Nature. 440 (2006) 
908-912. doi:10.1038/nature04645; b) Y.-L. Chang, Z.-H. Lu, White Organic Light-Emitting Diodes for Solid-State Lighting, Journal of Display Technology. 9 (2013) 459-468. doi:10.1109/JDT.2013.2248698; c) E.L. Williams, K. Haavisto, J. Li, G.E. Jabbour, ExcimerBased White Phosphorescent Organic Light-Emitting Diodes with Nearly 100 \% Internal Quantum Efficiency, Advanced Materials. 19 (2007) 197-202. doi:10.1002/adma.200602174.

[8] a) Y.-L. Chang, Z.B. Wang, M.G. Helander, J. Qiu, D.P. Puzzo, Z.H. Lu, Enhancing the efficiency of simplified red phosphorescent organic light-emitting diodes by exciton harvesting, Organic Electronics. 13 (2012) 925-931. doi:10.1016/j.orgel.2012.01.026; b) M.G. Helander, Z.B. Wang, J. Qiu, M.T. Greiner, D.P. Puzzo, Z.W. Liu, et al., Chlorinated Indium Tin Oxide Electrodes with High Work Function for Organic Device Compatibility, Science. 332 (2011) 944-947. doi:10.1126/science.1202992; c) H. Cao, H. Sun, Y. Yin, X. Wen, G. Shan, Z. Su, et al., Iridium (iii) complexes adopting 1, 2-diphenyl-1 H-benzoimidazole ligands for highly efficient organic light-emitting diodes with low-efficiency roll-off and non-doped feature, Journal of Materials Chemistry C. 2 (2014) 2150-2159; d) C.W. Lee, J.Y. Lee, Above 30\% External Quantum Efficiency in Blue Phosphorescent Organic Light-Emitting Diodes Using Pyrido[2,3- $b$ ]indole Derivatives as Host Materials, Advanced Materials. 25 (2013) 5450-5454. doi:10.1002/adma.201301091; e) J.-K. Bin, N.-S. Cho, J.-I. Hong, New Host Material for HighPerformance Blue Phosphorescent Organic Electroluminescent Devices, Advanced Materials. 24 (2012) 2911-2915. doi:10.1002/adma.201200972; f) D. Volz, M. Wallesch, C. Fléchon, M. Danz, A. Verma, J.M. Navarro, et al., From iridium and platinum to copper and carbon: new avenues for more sustainability in organic light-emitting diodes, Green Chem. 17 (2015) 19882011. doi:10.1039/C4GC02195A. 
[9] a) J. Kalinowski, V. Fattori, M. Cocchi, J.A.G. Williams, Light-emitting devices based on organometallic platinum complexes as emitters, Coordination Chemistry Reviews. 255 (2011) 2401-2425. doi:10.1016/j.ccr.2011.01.049; b) A. Poloek, C.-T. Chen, C.-T. Chen, High performance hybrid white and multi-colour electroluminescence from a new host material for a heteroleptic naphthyridinolate platinum complex dopant, Journal of Materials Chemistry C. 2 (2014) 1376. doi:10.1039/c3tc32394c; c) H. Fukagawa, T. Shimizu, H. Hanashima, Y. Osada, M. Suzuki, H. Fujikake, Highly Efficient and Stable Red Phosphorescent Organic Light-Emitting Diodes Using Platinum Complexes, Advanced Materials. 24 (2012) 5099-5103. doi:10.1002/adma.201202167; d) K. Li, G. Cheng, C. Ma, X. Guan, W.-M. Kwok, Y. Chen, et al., Light-emitting platinum(ii) complexes supported by tetradentate dianionic bis(N-heterocyclic carbene) ligands: towards robust blue electrophosphors, Chemical Science. 4 (2013) 2630. doi:10.1039/c3sc21822h; e) X. Wang, Y.-L. Chang, J.-S. Lu, T. Zhang, Z.-H. Lu, S. Wang, Bright Blue and White Electrophosphorescent Triarylboryl-Functionalized $\mathrm{C}^{\wedge} \mathrm{N}$-Chelate $\mathrm{Pt}(\mathrm{II})$ Compounds: Impact of Intramolecular Hydrogen Bonds and Ancillary Ligands, Advanced Functional Materials. (2013) n/a-n/a. doi:10.1002/adfm.201302871; f) Y.-C. Chiu, Y. Chi, J.-Y. Hung, Y.-M. Cheng, Y.-C. Yu, M.-W. Chung, et al., Blue to True-Blue Phosphorescent Ir ${ }^{\text {III }}$ Complexes Bearing a Nonconjugated Ancillary Phosphine Chelate: Strategic Synthesis, Photophysics, and Device Integration, ACS Applied Materials \& Interfaces. 1 (2009) 433-442. doi:10.1021/am800122n; g) Y.-L. Rao, D. Schoenmakers, Y.-L. Chang, J.-S. Lu, Z.-H. Lu, Y. Kang, et al., Bluish-Green BMes2-Functionalized PtII Complexes for High-Efficiency PhOLEDs: Impact of the BMes2 Location on Emission Color, Chemistry - A European Journal. 18 (2012) 11306-11316. doi:10.1002/chem.201201255. 
[10] X.-C. Hang, T. Fleetham, E. Turner, J. Brooks, J. Li, Highly Efficient Blue-Emitting Cyclometalated Platinum(II) Complexes by Judicious Molecular Design, Angewandte Chemie International Edition. 52 (2013) 6753-6756. doi:10.1002/anie.201302541.

[11] K. Kanakarajan, A.W. Czarnik, Synthesis and some reactions of hexaazatriphenylenehexanitrile, a hydrogen-free polyfunctional heterocycle with D3h symmetry, The Journal of Organic Chemistry. 51 (1986) 5241-5243.

[12] a) L. Xiao, S.-J. Su, Y. Agata, H. Lan, J. Kido, Nearly 100\% Internal Quantum Efficiency in an Organic Blue-Light Electrophosphorescent Device Using a Weak Electron Transporting Material with a Wide Energy Gap, Advanced Materials. 21 (2009) 1271-1274. doi:10.1002/adma.200802034; b) H. Sasabe, E. Gonmori, T. Chiba, Y.-J. Li, D. Tanaka, S.-J. Su, et al., Wide-Energy-Gap Electron-Transport Materials Containing 3,5-Dipyridylphenyl Moieties for an Ultra High-Efficiency Blue Organic Light-Emitting Device, Chemistry of Materials. 20 (2008) 5951-5953. doi:10.1021/cm801727d; c) N. Chopra, J.S. Swensen, E. Polikarpov, L. Cosimbescu, F. So, A.B. Padmaperuma, High efficiency and low roll-off blue phosphorescent organic light-emitting devices using mixed host architecture, Applied Physics Letters. 97 (2010) 033304. doi:10.1063/1.3464969.

[13] S.-J. Su, E. Gonmori, H. Sasabe, J. Kido, Highly Efficient Organic Blue-and WhiteLight-Emitting Devices Having a Carrier- and Exciton-Confining Structure for Reduced Efficiency Roll-Off, Advanced Materials. (2008) NA-NA. doi:10.1002/adma.200801375. [14] a) Y. Ohno, Spectral design considerations for white LED color rendering, Optical Engineering. 44 (2005) 111302. doi:10.1117/1.2130694; b) H. Ries, I. Leike, J. Muschaweck, Optimized additive mixing of colored light-emitting diode sources, Optical Engineering. 43 (2004) 1531-1536. 
[15] R. Meerheim, S. Scholz, S. Olthof, G. Schwartz, S. Reineke, K. Walzer, et al., Influence of charge balance and exciton distribution on efficiency and lifetime of phosphorescent organic light-emitting devices, Journal of Applied Physics. 104 (2008) 014510. doi:10.1063/1.2951960. [16] M.C. Gather, R. Alle, H. Becker, K. Meerholz, On the Origin of the Color Shift in WhiteEmitting OLEDs, Advanced Materials. 19 (2007) 4460-4465. doi:10.1002/adma.200701673.

[17] a) B. D’Andrade, S. Datta, S. Forrest, P. Djurovich, E. Polikarpov, M. Thompson, Relationship between the ionization and oxidation potentials of molecular organic semiconductors, Organic Electronics. 6 (2005) 11-20. doi:10.1016/j.orgel.2005.01.002; b) Lucia Leonat, Gabriela Sbârcea, Ioan Viorel Brânzoi, Cyclic voltammetry for energy levels estimation of organic materials, U.P.B. Sci. Bull. 75 (2013) 111.

[18] R.G. Kepler, J.C. Caris, P. Avakian, E. Abramson, Triplet excitons and Delayed Fluorescence in anthracene crystals., Phys. Rev. Lett. 10 (1963) 400-402. 